\title{
ÉTUDE DE L'ONCOMIRACIDIUM DE DIPLOZOON NIPPONICUM GOTO, 1891
}

\author{
Hapteur larvaire d'un type nouveau \\ pour le genre Diplozoon (Monogenea, Discocotylidae)
}

\author{
A. LAMBERT* ${ }^{*}$ et A. DENIS**
}

RÉSUMÉ. Diplozoon nipponicum Goto 1891 parasite de Cyprinus carpio est signalé pour la première fois en France et en Europe occidentale. Ce Monogène a été introduit avec des Carpes importées de Hongrie. L'oncomiracidium a été obtenu expérimentalement et étudié en détail (système excréteur, cellules ciliées, chétotaxie, hapteur). L'armature haptoriale est d'un type nouveau pour le genre Diplozoon : 2 paires de crochetons larvaires latéraux sont mis en évidence. Les implications taxonomiques et phylétiques découlant de ces observations sont discutées chez Diplozoon et chez les Discocotylidae. Il apparaît, en particulier, d'après l'étude du hapteur et de la structure de l'œuf, deux ensembles dont la signification taxonomique et évolutive sont à discuter chez Diplozoon.

\section{The oncomiracidium of Diplozoon nipponicum Goto, 1891. A new larval haptor for the genus Diplozoon (Monogenea, Discocotylidae).}

SUMMARY. Diplozoon nipponicum Goto 1891, a branchial parasite of Cyprinus carpio is mentioned for the first time in France and Western Europe. Carps, along with their Monogenea, were introduced from Hungary. After being obtained experimentally, the oncomiracidium is thoroughly studied (excretory system, ciliated cells, chaetotaxy, haptor). A new haptor for the genus Diplozoon is described: two pairs of larval lateral hooklets are present. The taxonomic and phylogenic implications of these observations are discussed for Diplozoon and Discocotylidae. Mainly as a result of the study of the haptor and the egg structure, it appears that two groups occur, the taxonomic and evolutive significance of which are to be discussed in Diplozoon.

Les premières descriptions de la larve de Diplozoon ont été faites par Zeller (1872) et Bychowsky (1957), mais c'est à Bovet $(1959,1967)$ que l'on doit des études détaillées, tant d'un point de vue morphologique que biologique.

* Laboratoire de Parasitologie comparée, Université des Sciences et Techniques du Languedoc, place EugèneBataillon, F 34060 Montpellier Cedex.

** Laboratoire d'Ichthyologie et de Parasitologie générale, Université des Sciences et Techniques du Languedoc, place Eugène-Bataillon, F 34060 Montpellier Cedex.

Accepté le 8 juillet r982. 
Déjà, par sa larve, le genre Diplozoon se singularise des autres Monogènes Polyopisthocotylea : c'est la seule dont le hapteur possède un minimum de pièces haptoriales : une paire de pinces, une paire de crochets à fléau postérieurs (crochets I). Cette armature haptoriale a été interprétée comme illustrant une ontogénèse avancée puisque la larve, dès l'éclosion, possède une paire de pinces qui, chez les autres Polyopisthocotylea n'apparaît en général qu'après la fixation de l'oncomiracidium, au cours du développement post-larvaire. Cependant, on connaît quelques espèces dont le hapteur larvaire est déjà armé d'une paire de pinces : Polylabris tubicirrus (Paperna et Kohn, 1964), Microcotyle mugilis Vogt 1878, Discocotyle sagittata (Leuckart, 1842) et Microcotyle mormyri Lorenz 1878 (voir Euzet et Cauwet, 1967 ; Euzet et Combes, 1969 ; Owen, 1970 ; Ktari, 1971). Sterba (1957) signale même chez la larve de Diplozoon tetragonopterini, Sterba, 1957 une deuxième paire de pinces.

Si une ontogénèse plus ou moins avancée, au stade de l'éclosion permet de rendre compte de l'existence d'une - ou deux — paires de pinces remplaçant les crochetons latéraux III de l'oncomiracidium, il est plus difficile d'expliquer l'absence, à ce même stade du cycle, des autres crochetons embryonnaires chez Diplozoon.

L'utilisation d'imprégnations argentiques a permis de mieux connaître l'équipement sensoriel et locomoteur de la larve (Euzet et Lambert, 1971, 1974 ; Khotenovski, $1975,1977)$ chez plusieurs espèces de Diplozoon. Nous complétons la description de l'oncomiracidium de Diplozoon nipponicum (Goto 1891) déjà ébauchée par Kamegai (1968, 1972).

\section{Matériel et méthodes}

Nous avons récolté, sur les branchies de la Carpe, Cyprimus carpio, provenant d'une pisciculture du Sud-est de la France, des couples adultes de Diplozoon nipponicum Goto 1891. Tous les caractères morphométriques de l'adulte et des œufs correspondent à ceux donnés par Kamegai et coll. (1966) et Kamegai (1968) qui a redécrit cette espèce initialement signalée sur Carassius carassius au Japon.

Diplozoon nipponicum est donc signalé pour la première fois en France et en Europe occidentale. Les Carpes parasitées provenaient de Hongrie où l'on doit retrouver ce parasite.

Le cycle de cette espèce est susceptible de se réaliser en Camargue (Sud-est de la France) : après avoir isolé les poissons parasités dans un aquarium, nous avons pu récolter les œufs des couples adultes sur un tulle fin, tendu sur le siphon d'aspiration de la pompe filtrant l'eau du bac d'élevage. Ces œufs sont mis en incubation dans de petits cristallisoirs dont l'eau, filtrée sur " Millipore ", est renouvellée quotidiennement. A la température du laboratoire $\left(22^{\circ} \mathrm{C}\right)$ une semaine est nécessaire pour obtenir les oncomiracidiums.

L'étude de la morphologie générale est faite sur des larves observées in vivo entre lame et lamelle (système excréteur, hapteur). Les imprégnations argentiques ont été réalisées selon la méthode précédemment décrite (Lambert, 1980) : imprégnation directe par une solution d'Ag $\mathrm{NO}_{3}$ à $6 \%$, révélation à la lumière solaire $(5 \mathrm{mn})$, 
rinçage et montage direct dans une gomme au chloral (Liquide de Faure). Les larves sont immédiatement dessinées après leur montage, à la chambre claire (Wild M20), les détails révélés par l'imprégnation se dégradant avec le temps.

La nomenclature des pièces haptoriales est celle adoptée à ICOPA IV (Varsovie, 1978) selon les propositions de Llewellyn (1963)*.

\section{Résultats}

L'allure de la larve est celle déjà décrite par les auteurs précédents. Son comportement dans l'eau coïncide avec les observations de Bovet (1959-1967). Si des similitudes existent dans l'organisation des cellules ciliées, de la chétotaxie et de l'appareil excréteur de l'oncomiracidium de Diplozoon nipponicum, des différences profondes sont observées dans l'armature du hapteur.

\section{1 - Le système excréteur}

L'organisation générale du système excréteur correspond à celle décrite par Bovet (1967) et Euzet et Lambert (1971). La formule excrétrice est :

ce qui correspond à :

$$
2[(2+2)+(2+2)]=16
$$

- 2 paires de protonéphridies céphaliques

-2 paires de protonéphridies pharyngiennes

-2 paires de protonéphridies pleurales

-2 paires de protonéphridies haptoriales

\section{2 - Les cellules ciliées ( $f i g .1$ )}

La couverture ciliée se compose de 41 cellules réparties en 6 groupes, comme chez les autres Diplozoon décrits :

- 1 groupe frontal de 4 cellules : 2 sont impaires et médianes et 2 latérales,

- 2 groupes antérieurs composés de chaque côté par 6 cellules latéro-ventrales,

- 2 groupes médians composés de chaque côté par 6 cellules latéro-ventrales,

- 1 groupe postérieur de 13 cellules : 10 cellules en couronne autour de la région postérieure et 3 petites cellules terminales.

\section{3 - Les organes sensoriels}

a - Les sensilles (fig. 1)

Les récepteurs sensoriels tégumentaires se répartissent de la manière suivante :

- dorsalement, par hémicorps :

- 1 groupe oculaire de 3 sensilles dans la concavité de chaque tache oculaire,

* Les crochets sont numérotés en chiffre romain du plus postérieur au plus antérieur, à l'exception des hamuli. Ainsi les crochets I représentent les crochets postérieurs à fléau; les crochets II, les postéro-latéraux et les crochets III à VI, les latéraux, numérotés d'arrière en avant. 
- 1 groupe post-oculaire de 3 sensilles alignées longitudinalement,

- 1 groupe dorso-latéral de 6 sensilles,

- 1 groupe postérieur de 4 sensilles.
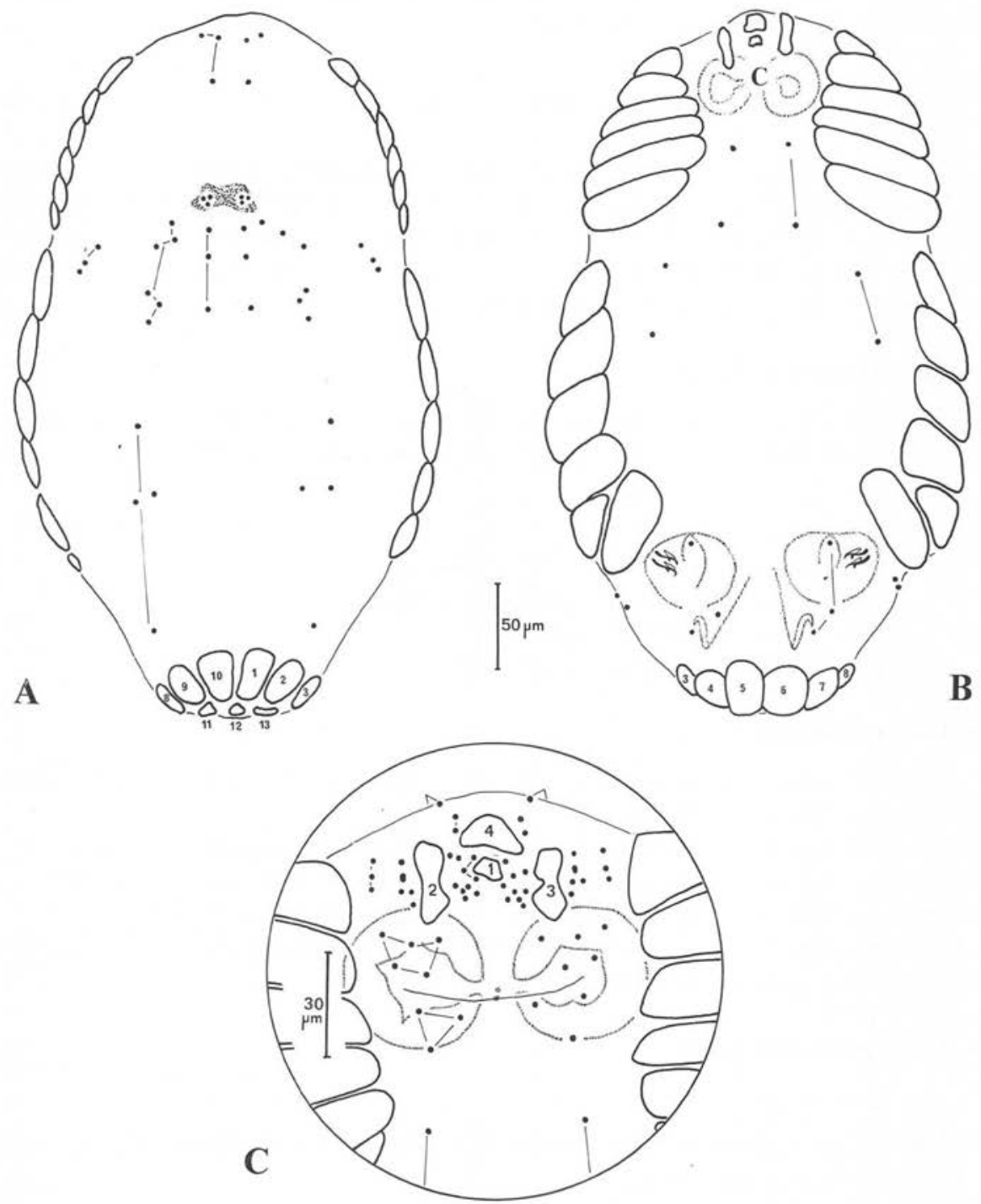

FIG. r. - Diplozoon nipponicum Goto I891 : cellules ciliées et chétotaxie de l'oncomiracidium (dessin à la chambre claire d'un spécimen). A : face dorsale ; B : face ventrale ; C : cadre buccal et région frontale (détail).

Les cellules ciliées haptoriales sont numérotées de $\mathrm{I}$ à $\mathrm{I}_{3}$; celles du groupe frontal de $\mathrm{I}$ à 4 . Les sensilles sont groupées d'après la description donnée dans le texte. 
- ventralement, par hémicorps :

- 1 groupe antérieur de 2 sensilles,

- 1 groupe médian de 2 sensilles,

- 1 groupe haptorial de 3 sensilles.

- latéralement, par hémicorps :

- 1 groupe de 3 sensilles au niveau du pore excréteur,

- 1 groupe de 2 sensilles en arrière des cellules ciliées médianes.

- groupe frontal complexe, par hémicorps :

- 1 groupe de 3 sensilles frontales,

- 1 groupe de 2 sensilles contre la cellule frontale dorsale,

- 1 groupe de 3 sensilles contre la cellule frontale médiane,

- 1 groupe de 5 sensilles à la base de la cellule frontale médiane

- 1 groupe de 5-6 sensilles autour de chaque cellule frontale latérale,

- 1 groupe de 3 sensilles frontales latérales.

- groupe buccal, par hémicorps :

Il y a 3 sensilles sur la lèvre inférieure et 5 sur la lèvre supérieure.

Il semble enfin exister 2 sensilles buccales internes médianes impaires.

La chétotaxie s'avère donc semblable à celle des autres espèces de Diplozoon, malgré quelques différences de détail.

b - Les photorécepteurs

En microscopie optique, sur le vivant, les deux types de photorécepteurs : photorécepteurs médians pigmentés et photorécepteurs latéraux non pigmentés (Kearn, 1978) sont nettement visibles. Une relation très nette ( fig. 2) existe entre les deux ; c'est un " fin cordon » matérialisé par des granules pigmentaires semblables à ceux des taches oculaires médianes. Kearn, 1978, dit observer cette connexion en contraste de phase mais ne la figure pas sur le schéma donné. A l'évidence, d'après les

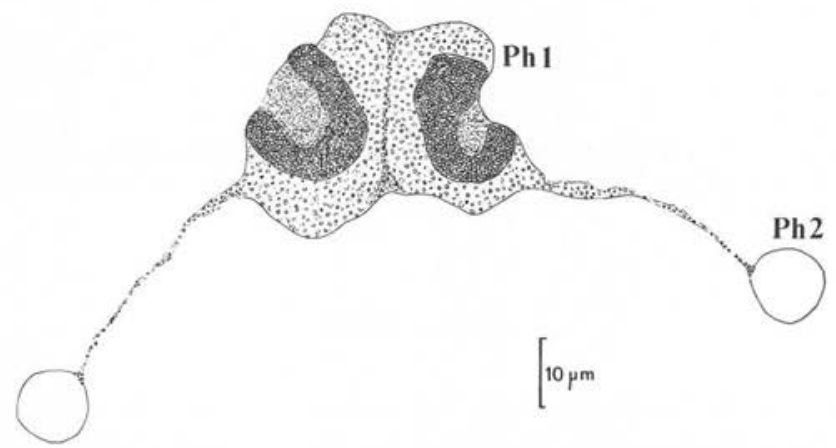

FIG. 2. - Diplozoon nipponicum Goto I891 : dessin à la chambre claire des photorécepteurs de l'oncomiracidium (vue dorsale). $\mathrm{Ph}$. I : photorécepteurs médians pigmentés; $\mathrm{Ph} .2$ : photorécepteurs latéraux non pigmentés. 
remarques de cet auteur et nos propres observations faites sur les Diplozoon appartenant à d'autres espèces, cette liaison interoculaire habituellement discrète est particulièrement nette et développée chez Diplozoon nipponicum.

\section{4 - Le hapteur (fig. 3)}

L'oncomiracidium de Diplozoon nipponicum présente l'armature haptoriale suivante, par hémicorps :

- 1 crochet $\mathrm{I}$ : manche 50 à $55 \mu \mathrm{m}$, lame 23 à $25 \mu \mathrm{m}$

- 1 pince : $55 \mu \mathrm{m} / 40 \mu \mathrm{m}$

- 2 crochetons de 15 à $17 \mu \mathrm{m}$ : ce sont les crochets IV et V si nous considérons que la paire de pince remplace les crochets III.

- (Les crochets II et VI sont absents).

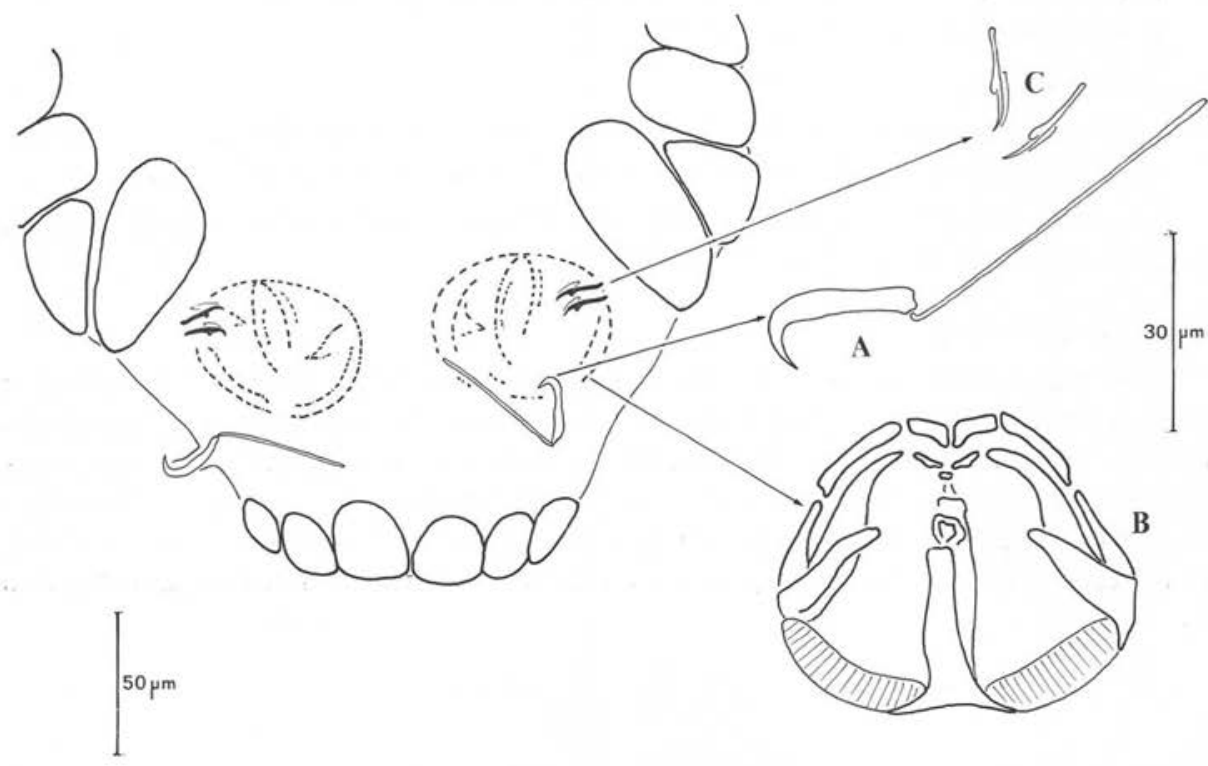

FIG. 3. - Diplozoon nipponicum Goto I891 : détail de la région haptoriale de l'oncomiracidium. $\mathrm{A}$ : crochets I; B : pinces; C : crochetons IV et V (dessins à la chambre claire).

\section{Discussion}

Ces résultats nous amènent à deux types de remarques : les premières sont relatives à la valeur phylétique que l'on peut accorder à diverses structures de l'oncomiracidium, les secondes reposent le problème de la spécificité parasitaire, de la spéciation du genre Diplozoon et de toute la systématique des Discocotylidae. 
1 - L'étude comparée des oncomiracidiums des espèces du genre Diplozoon décrites jusqu'à présent met en évidence deux types de structures larvaires :

- Les structures larvaires stables:

Il s'agit du système excréteur, de la chétotaxie et des cellules ciliées.

Le système excréteur correspond à la formule $2[(2+2)+(2+2)]=16$ chez Diplozoon paradoxum (chez Abramis brama), Diplozoon gracile (chez Barbus meridionalis, Gobio gobio, Phoxinus phoxinus, Telestes soufia) Euzet et Lambert, 1974; Diplozoon homoion (chez Rutilus rutilus), observation personnelle non publiée et chez Diplozoon nipponicum (chez Cyprinus carpio), présent travail.

La couverture ciliée est identique dans tous les cas décrits à savoir : 4 cellules frontales, $2 \times 6$ cellules antérieures, $2 \times 6$ cellules médianes et 13 cellules haptoriales soit 41 cellules. Cette distribution pouvant se formuler de la manière suivante :

$$
4+(2 \times 6)+(2 \times 6)+13=41 .
$$

Ceci s'observe chez Diplozoon gracile, D. paradoxum, D. homoion, D. markewitschi, D. rutili et D. megan (Euzet et Lambert, 1974; Khotenovski, 1975 et 1977). Il en est de même pour $D$. nipponicum.

La chétotaxie de ces espèces est très semblable. On retrouve les mêmes groupes de sensilles, et le même nombre de sensilles dans chaque groupe.

Quelques petites variations peuvent exister, permettant par exemple de séparer D. paradoxum de D. gracile (Euzet et Lambert, 1974) ; mais ces variations n'affectent que la disposition relative des cellules ciliées antérieures et des sensilles ventrales, pas leur nombre. De même, la disposition des cellules ciliées médianes chez D. nipponicum diffère légèrement de celle des autres espèces où la cellule précédant la plus postérieure de ce groupe est passée dorsalement. Chez D. nipponicum cette cellule latéro-dorsale est encore intercalée entre la cellule la plus postérieure du groupe et les autres. A noter que cette disposition se retrouve chez l'oncomiracidium de Discocotyle sagittata ( fig. 4) (Owen, 1970). Nous avons par ailleurs (Lambert, 1980) démontré, en étudiant ces structures argyrophiles chez d'autres oncomiracidiums de Monogènes marins que, s'il existe quelques variations interspécifiques voire intergénériques, ce qui est fondamental, c'est au contraire l'existence d'un plan d'organisation commun de la chétotaxie et des cellules ciliées.

\section{- Les structures larvaires variables}

Le hapteur larvaire de $D$. nipponicum s'avère fondamentalement différent de celui de toutes les autres espèces de ce genre. C'est le seul à posséder deux paires de crochetons latéraux. Cette observation amène deux réflexions.

Ceci confirme, s'il en était besoin, les affinités phylétiques existant entre Diplozoon et les autres Discocotylidae (Octomacrum, Neodiscocotyle, Discocotyle). D. nipponicum fait figure de "maillon intermédiaire " entre le hapteur de Discocotyle (3 paires de crochetons) et celui des autres espèces de Diplozoon (pas de crochetons).

On peut donc imaginer à partir d'un hapteur larvaire commun ancestral, une évolution qui s'est manifestée au niveau de cet organe de la manière suivante : 

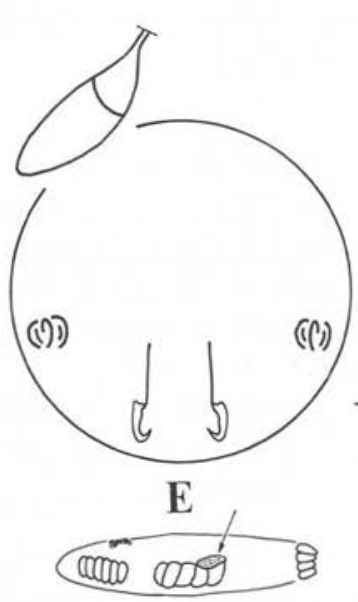

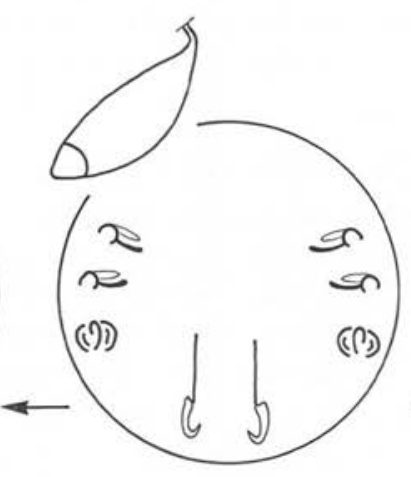

D

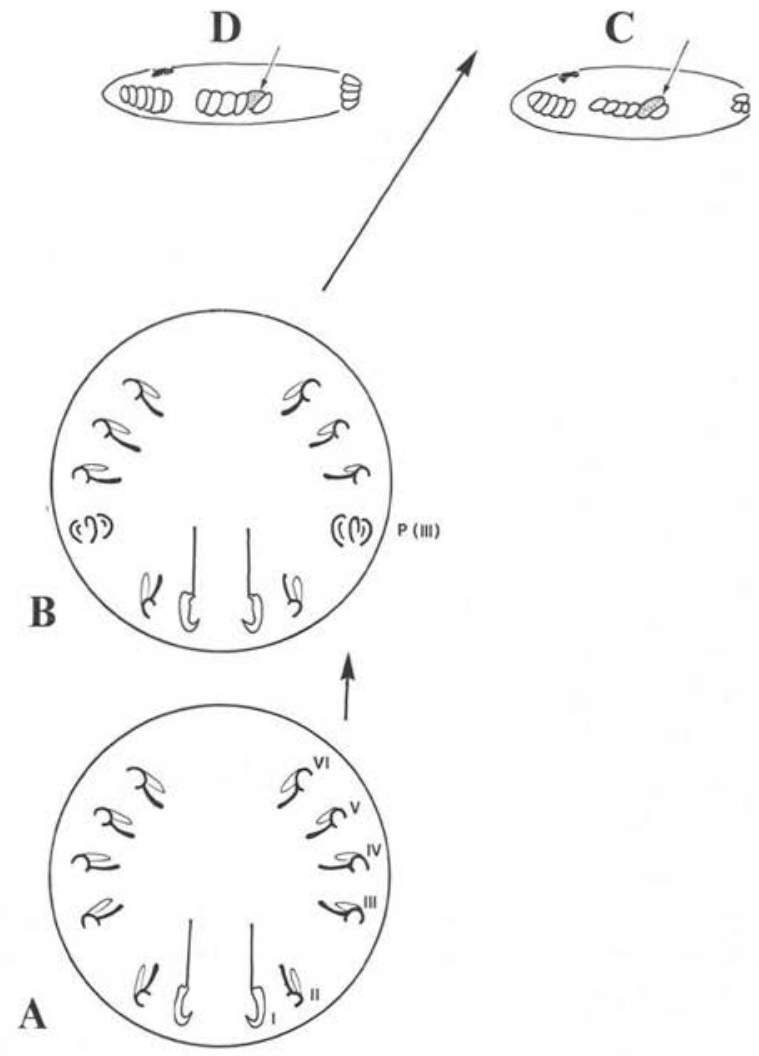

FIG. 4. - Evvolution du hapteur des Discocotylidae. A et B : hapteurs hypothétiques ancestraux ; C: Discocotyle sagittata ( ()$; D$ : Diplozoon nipponicum ; E : Diplozoon paradoxum, D. homoion, D. gracile, etc. Les œufs ainsi que les cellules ciliées sont schématisés pour chacune de ces espèces. Dans chaque groupe de cellules ciliées médiane une flèche indique les variations dans la position de la $5^{\mathrm{e}}$ cellule.

(1) La paire de crochetons médians dégénérés décrits par Owen (1970) ne sont pas ici représentés, le problème de leur homologie n'étant pas résolu. 
ontogénèse précoce avec apparition à l'éclosion d'une paire de pince en remplacement des crochetons III et disparition des crochetons II. Par la suite, tous les autres crochetons larvaires (IV à VI) régressent sans être remplacés, à ce moment là par une paire de pinces.

Quel que soit le type de relation phylétique que l'on peut imaginer entre ces 3 types de hapteur larvaire, la tendance évolutive nette qui apparaît, c'est la spécialisation du hapteur larvaire par disparition de crochetons latéraux. Dans cette optique D. nipponicum serait le Diplozoon le plus primitif.

Il est aussi intéressant de noter que c'est précisément la seule espèce de ce genre qui possède un œuf de type particulier avec le filament opposé à l'opercule. Cette particularité prend toute sa signification à la suite des remarques précédentes : la structure de l' œuf correspondrait ici à un critère dont on a sous-estimé jusqu'ici l'importance taxonomique et phylétique. C'était oublier que la structure de l'œuf est le résultat d'une série de phénomènes embryologiques et morphogénétiques qui interviennent dans l'ootype. Ces phénomènes sont certes mal connus mais il $\mathrm{y}$ a tout lieu de penser qu'ils ont un rôle fondamental à jouer dans l'élaboration de la coque, du filament et donc de la polarité de l'œuf.

2 - Les conséquences de ces observations sur le plan taxonomique sont importantes.

La systématique des Diplozoon a été toujours confuse et les travaux expérimentaux de Bovet (1967) ont eu le mérite de poser le problème de la spéciation du genre en terme biologique. Tout récemment, Khotenovsky (1981) propose de regrouper toutes les espèces de ce genre dans une famille nouvelle : les Diplozoidae*, elle-même subdivisée en 2 sous-familles. Le genre Diplozoon éclate en 6 genres nouveaux : Neodiplozoon Tripathi 1960, Afrodiplozoon Khotenovsky 1981, Paradiplozoon Achmerov 1974, Diplozoon Nordmann 1832, Inustiatus Khotenovsky 1978 et Sindiplozoon Khotenovsky 1981.

Cette proposition nous semble prématurée. Pourquoi la baser sur des critères morphologiques des adultes alors qu'il existe au niveau de l'œuf et de la larve des différences importantes?

Une taxonomie rationnelle ne pourra intervenir que lorsque nous connaîtrons les oncomiracidiums des autres Diplozoon et des Discocotylidae; en particulier les genres Neodiplozoon, Octomacrum et Neodiscocotyle. On peut imaginer que la larve de Discocotyle sagitatta décrite par Owen (1970) est particulière : c'est en effet le seul Discocotylidae de Salmonidae alors qu'Octomacrum et Neodiscocotyle (comme les Diplozoon) sont parasites de Cyprinidae et Catostomidae. L'essentiel de l'évolution des Discocotylidae s'étant réalisée chez ces Cypriniformes, c'est à partir de l'étude des larves de spécimens récoltés sur ces hôtes que nous aurons peut-être une idée plus précise de l'évolution de ces Monogènes. Ceci est d'autant plus intéressant qu'ils constituent l'essentiel des rares Polyopisthocotylea existant en eau douce.

* Cette dénomination est d'ailleurs incorrecte pour une famille ; ce devrait être Diplozoonidae. 
Remerciements : Ce travail a été réalisé dans le cadre de l'ERA CNRS (915) "Biologie et écologie des Plathelminthes parasites ", et du Centre de Parasitologie et Pathologie Comparée de l'Université des Sciences et Techniques Languedoc (Montpellier).

Nous remercions Monsieur le Professeur Louis Euzet pour son aide et ses critiques au cours de la réalisation du manuscrit.

\section{BIBLIOGRAPHIE}

Bovet J. : Observations sur l'œuf et l'oncomiracidium de Diplozoon paradoxum V. Nordmann 1832. Bull. Soc. Neuchâtel. Sci. Nat., 1959, 82, 231-245.

Bovet J. : Contribution à la morphologie et à la biologie de Diplozoon paradoxum V. Nordmann 1832. Bull. Soc. Neuchatel. Sci. Nat., 1967, 90, 63-159.

Bychowsкy B. E. : Mogenetic trematodes, their systematics and phylogeny (en russe) Akad. Nauk SSSR 1957, 509 p. English Transl. AIBS. Washington by W. Hargis, 196r.

Euzet L., CAUWET A. : Polylabris diplodi n.g. n.sp. (Monogenea Microcotylidae) parasite de Téléostéens du genre Diplodus (Sparidae). Bull. Mus. Nat. Hist. Nat., 1967, 39, 21 3-220.

Euzet L., Combes C. : Contribution à l'étude des Microcotylidae (Monogenea) parasites de Mugil cephalus L. (Téléostei). Parasitol. Sb. Zool. Inst. Akad. Nauk SSSR I969, 24, 9I-ro5.

Euzet L., Lambert A. : Compléments à l'étude de la larve de Diplozoon paradoxum V. Nordmann I832 (Monogenea). Ann. Parasitol. Hum. Comp., 1971, 46, 675-684.

Euzet L., Lambert A. : Chétotaxie comparée des larves de Diplozoon paradoxum V. Nordmann I832 et de Diplozoon gracile Reichenbach-Klinke I961 (Monogenea). Bull. Soc. Zool. France, 1974, 99, 307-314.

Kamegai S., Ichihara A., Kato K., Nonobe H., Machida M. : Diplozoon nipponicum Goto I89I Part I. Morphological observations on the worms obtained from Cyprinus carpio. Meguro. Parasit. Museum, 1966, I-16.

Kamegar S. : On Diplozoon nipponicum Goto 1891 Part 2. The distribution in Japan and the developmental observation. Res. Bull. Meguro. Parasit. Museum, 1968, 2, I-8.

Kamegar S. : Observation on the development of the oncomiracidium of Diplozoon nipponicum Goto 1891. Jap. J. Parasit., 1972, 21, suppl. 81.

Kearn G. C. : Eyes with and without pigments shields in the oncomiracidium of the Monogenea Parasite Diplozoon paradoxum. Z. Parasitenk., 1978, 157, 35-47.

Khotenovsky I. A. : On the structure of eggs and larvae of some Diplozoon species (en russe). Parazitologija URSS, 1975, 9, 17-27.

KнотеNovsky I. A. : On the structure of eggs and larvae of Diplozoon megan (Monogenea, Diplozoonidae) (en russe). Parazitologija URSS, 1977, $11,456-458$.

КнотеNovSKY I. A. : Taxonomy and phylogeny of Monogeneans of the families Diplozoidae and Discocotylidae (Monogenea) (en russe). Parasitol. Sbor., 1981, 30, 166-178.

KTARI M. H. : Recherches sur la reproduction et le développement de quelques Monogènes (Polyopisthocotylea) parasites de Poissons marins. Thèse doctorat d'État, 1971, Université des Sciences et Techniques du Languedoc, Montpellier, $\mathrm{n}^{\circ}$ CNRS AO 6332, $327 \mathrm{p}$.

LAMBert A. : Oncomiracidiums et phylogénèse des Monogenea. $2^{\mathrm{e}}$ partie : Structures argyrophyles des oncomiracidiums et phylogénèse des Monogenea. Ann. Parasitol. Hum. Comp., I980, 55, $28 \mathrm{I}-325$.

Llewellyn J. : Larvae and larval development of Monogeneans. Adv. Parasitol., 1963, 1, 287-326.

OwEN J. L. : The oncomiracidium of the monogenean Discocotyle sagittata. Parasitology, 1970, $61,279-292$.

Sterba G. : Zur morphologie und Biologie der Gattung Diplozoon. Zool. Anzeiger, I957, I s8, I8II96.

ZELLER E. : Untersuchungen über die Entwicklung des Diplozoon paradoxum. Z. Zool., I872, 22, I68-180. 